\title{
Synthesis of novel 3, 5, 6-trisubstituted triazine derivatives and their biological activity evaluation as potential antitumor and anti-inflammatory agents
}

\author{
Leyla Yurttaş ${ }^{1}$, Zafer Şahin², Gülşen Akalın Çiftçi ${ }^{3}$, \\ Halide Edip Temel ${ }^{3}$, Şeref Demirayak² \\ ${ }^{1}$ Anadolu University, Faculty of Pharmacy, Department of Pharmaceutical Chemistry, Eskişehir, Turkey \\ ${ }^{2}$ Medipol University, School of Pharmacy, Department of Pharmaceutical Chemistry, İstanbul, Turkey \\ ${ }^{3}$ Anadolu University, Faculty of Pharmacy, Department of Biochemistry, Eskişehir, Turkey
}

\begin{abstract}
In this study, new 3, 5, 6-trisubstituted 1, 2, 4-triazine derivatives (1-9) were synthesized and their structures were determined by using NMR, IR and Mass spectroscopic methods. In vitro antitumor activities against $\mathrm{MCF}-7$ breast adenocarcinoma and C6 rat glioma cell lines were evaluated via MTT colorimetric assay. Among the compounds, compound $4\left(\mathrm{IC}_{50}=21.0 \mu \mathrm{g} / \mathrm{mL}\right)$ was found as the most active one against $\mathrm{C} 6$ cell line, whereas compound $\mathbf{5}\left(\mathrm{IC}_{50}=9.5 \mu \mathrm{g} / \mathrm{mL}\right)$ was found the most potent compound against MCF-7 cell line and both of compounds had higher activity than cisplatin in their line. Furthermore, $\mathrm{IC}_{50}$ value of compound 6 was found as $26.0 \mu \mathrm{g} / \mathrm{mL}$ against C6 which was very close to cisplatin potency $\left(\mathrm{IC}_{50}=23.5 \mu \mathrm{g} / \mathrm{mL}\right.$ ). Besides, all compounds were tested to determine their lipoxygenase (LOX) inhibitory activity. Compounds $\mathbf{1}$ and $\mathbf{6}$ showed LOX inhibition with percentages of $43.35 \%$ and $38.79 \%$ at $100 \mu \mathrm{g} / \mathrm{mL}$ concentration, respectively. The obtained results on cell lines inspire to synthesise new and more potent molecules compounds as anticancer agents.
\end{abstract}

Keywords: Triazine, cytotoxicity, antitumor, lipooxygenase (LOX) inhibition

\section{INTRODUCTION}

Together cardiovascular diseases, cancer is one of the common causes of death. By the discovery of mechanisms of cancer, efforts focused on different targets to treat disease. Besides the new techniques like hyperthermia, photodynamic therapy and stem cell transplation; old techniques radiotherapy, hormonal and non-hormonal chemotherapy, immunotherapy and surgery methods is still be-

*Corresponding author: Leyla YURTTAŞ

E-mail address: Iyurttas@anadolu.edu.tr 
ing used ${ }^{1}$. In chemotherapy, alkylation agents, mitotic inhibitors and antimetabolites are used either alone or in combination with each other. In simple terms, a selective drug must be active on cancer cells but inactive in normal cells. But the differences between normal and cancer cells is quite little and not qualitative so that its hard to find an exploitable mechanism².

Purine and pyrimidine analog drugs such as 5-fluorouracil and 6-mercaptopurine have been widely used in succesful treatment of cancer disease ${ }^{3}$. Many nitrogen containing heterocylic compounds such as 1,2,4-triazines have been constitutes medicinal chemists' interest area in cancer theraphy ${ }^{4,5}$. The potential anticancer-cytostatic effects of 1,2,4-triazine derivatives were widely studied and reported to have promising activity ${ }^{6-12}$.

5-Lipoxygenase (5-LOX) is a crucial enzyme which catalyses biosynthesis of leukotrienes in the arachidonic acid (AA) cascade. Variable leukotriene levels were reported in different diseases like cardiovascular diseases and certain types of cancer as well as asthma, allergic rhinitis ${ }^{13}$. In particular, 5 -LOX have been found to be up-regulated in many cancer cell lines, which results in promoting the development of carcinogenesis. Inhibition of LOX enzyme could be a subsidiary mechanism for down-regulation of tumors accordingly this approach enables a rational concept for the design of more effective antitumor agents ${ }^{14}$.

Accordingly, we aimed to synthesize 1-[4-(5,6-bis-(4-substituted phenyl)-1,2,4triazin-3-yl)piperazin-1-yl]-2-[(1H-(benz)imidazol/thiazole/oxazole-2-yl)thio] ethan-1-one derivatives (1-9) for evaluating anticancer activity by following a study reported before a number of derivatives with similar structures ${ }^{15}$. The structure elucidation was carried out by spectroscopic techniques and in vitro anticancer activities of compounds were evaluated using MTT technique on MCF-7 and C6 tumor cell lines. The lipooxygenase (LOX) enzyme inhibitory activiy of the compounds were also studied.

\section{METHODOLOGY}

\section{Chemistry}

\section{Synthesis and characterization}

All needed chemicals were purchased from Sigma-Aldrich (Sigma-Aldrich Corp., St. Louis, MO, USA). All melting points (m.p.) were determined by MP9o digital melting point apparatus (Mettler Toledo, $\mathrm{OH}$ ) and were uncorrected. All the reactions were monitored by thin-layer chromatography (TLC) using Silica Gel 60 F254 TLC plates (Merck KGaA, Darmstadt, Germany). Spectroscopic data were recorded with the following instruments: a Bruker Tensor 27 IR spectrophotometer; ${ }^{1} \mathrm{H}$ NMR (nuclear magnetic resonance) Bruker DPX- 300 FT-NMR 
spectrometer, ${ }^{13} \mathrm{C}$ NMR, Bruker DPX $75 \mathrm{MHz}$ spectrometer (Bruker Bioscience, Billerica, MA, USA); M+1 peaks were determined by Shimadzu LC/MS ITTOF system (Shimadzu, Tokyo, Japan). The synthesis of three intermediate products, 2-chloro-1-[4-(5,6-bis-(4-substituted phenyl)-1,2,4-triazin-3-yl)piperazin-1-yl] ethanones (IIIa-c) was carried out by the following study of Demirayak and coworkers ${ }^{15}$.

\section{General procedure for the synthesis of the final compounds (1-9)}

Equal moles of halogenated compounds (IIIa-c), 2-mercapto(benz)imidazole/ thiazole/oxazole and potassium carbonate were stirred in acetone for $5 \mathrm{~h}$. At the end of the reaction, the solvent was evaporated and the residue was treated with water. The obtained precipitation was filtered and it recrystallized from ethanol after dryness.

To realize the characterization and identification of compounds, Infrared${ }^{1} \mathrm{H}-\mathrm{NMR},{ }^{13} \mathrm{C}-\mathrm{NMR}$ and mass spectroscopy methods were used. Melting points were determined by using stuart melting apparatus. Yields and elemental analysis also calculated for each compound.

\section{1-[4-(5,6-Bis-(4-methylphenyl)-1,2,4-triazin-3-yl)piperazin-1-yl]-2- [(1H-benzimidazol-2-yl)thio]ethan-1-one (1):}

75 \% yield; mp $248^{\circ} \mathrm{C}$. IR v $v_{\max }\left(\mathrm{cm}^{-1}\right): 1639(\mathrm{C}=\mathrm{O}), 1684$ (amide $\left.\mathrm{C}=\mathrm{O}\right), 1525-1379$ $(\mathrm{C}=\mathrm{C}, \mathrm{C}=\mathrm{N}), 1269-1051(\mathrm{C}-\mathrm{N}) .{ }^{1} \mathrm{H}-\mathrm{NMR}\left(300 \mathrm{MHz}, \mathrm{DMSO}-d_{6}, \mathrm{ppm}\right) \delta 2.31(\mathrm{~s}$, $6 \mathrm{H}, \mathrm{CH}_{3}$ ), 3.67 (brs, $2 \mathrm{H}$, piperazine $\mathrm{CH}_{2}$ ), 3.78 (brs, $2 \mathrm{H}$, piperazine $\mathrm{CH}_{2}$ ), 3.92 (brs, $2 \mathrm{H}$, piperazine $\mathrm{CH}_{2}$ ), 4.04 (brs, $2 \mathrm{H}$, piperazine $\mathrm{CH}_{2}$ ), $4.5 \mathrm{O}\left(\mathrm{s}, 2 \mathrm{H}, \mathrm{CH}_{2} \mathrm{CO}\right.$ ), 7.09-7.13 (m, 2H, Ar-H), 7.17 (d, J=8.07 Hz, 2H, Ar-H), 7.27 (d, J=8.13 Hz, $2 \mathrm{H}, \mathrm{Ar}-\mathrm{H}$ ), 7.37 (d, J=8.13 Hz, 2H, Ar-H), 7.44 (brs, 2H, Ar-H), 12.58 (s, $1 \mathrm{H}$, $\mathrm{NH}) .{ }^{13} \mathrm{C}-\mathrm{NMR}\left(75 \mathrm{MHz}, \mathrm{DMSO}-d_{6}, \mathrm{ppm}\right) \delta 21.28\left(\mathrm{CH}_{3}\right), 21.38\left(\mathrm{CH}_{3}\right), 35.59$ $\left(\mathrm{COCH}_{2}\right), 41.78\left(\mathrm{CH}_{2}\right), 43.38\left(\mathrm{CH}_{2}\right), 43.68\left(\mathrm{CH}_{2}\right), 45.53\left(\mathrm{CH}_{2}\right), 121.83,129.21$, 129.35, 129.41, 129.87, 133.76, 133.96, 138.11, 140.68, 148.95, 150.22, 155.64, 159.60, $166.46(\mathrm{C}=\mathrm{O})$. For $\mathrm{C}_{30} \mathrm{H}_{29} \mathrm{~N}_{7} \mathrm{OS} \operatorname{HRMS}(\mathrm{m} / \mathrm{z})$ : $[\mathrm{M}+\mathrm{H}]^{+}$calcd: 534.67 ; found 536.22 .

\section{1-[4-(5,6-Bis-(4-methylphenyl)-1,2,4-triazin-3-yl)piperazin-1-yl]-2- [(1H-benzoxazol-2-yl)thio]ethan-1-one (2):}

$72 \%$ yield; mp $135{ }^{\circ} \mathrm{C}$. IR $v_{\max }\left(\mathrm{cm}^{-1}\right): 1651(\mathrm{C}=\mathrm{O}), 1533-1381(\mathrm{C}=\mathrm{C}, \mathrm{C}=\mathrm{N}), 1219$ 1068 (C-N, C-O). ${ }^{1} \mathrm{H}-\mathrm{NMR}\left(300 \mathrm{MHz}, \mathrm{DMSO}-d_{6,} \mathrm{ppm}\right) \delta 2.32\left(\mathrm{~s}, 6 \mathrm{H}, \mathrm{CH}_{3}\right), 3.69$ (brs, $2 \mathrm{H}$, piperazine $\mathrm{CH}_{2}$ ), 3.77 (brs, $2 \mathrm{H}$, piperazine $\mathrm{CH}_{2}$ ), 3.94 (brs, 2H, piperazine $\mathrm{CH}_{2}$ ), 4.06 (brs, 2H, piperazine $\mathrm{CH}_{2}$ ), 4.66 (s, 2H, $\mathrm{CH}_{2} \mathrm{CO}$ ), 7.17 (d, J= 7.71 $\mathrm{Hz}, 4 \mathrm{H}, \mathrm{Ar}-\mathrm{H}$ ), 7.26-7.35 (m, 6H, Ar-H), 7.63-7.7.68 (m, 2H, Ar-H). ${ }^{13} \mathrm{C}-\mathrm{NMR}(75$ MHz, DMSO- $d_{6}$ ppm) $\delta 21.29\left(\mathrm{CH}_{3}\right), 21.38\left(\mathrm{CH}_{3}\right), 36.98\left(\mathrm{COCH}_{2}\right), 41.89\left(\mathrm{CH}_{2}\right)$, 
$43.34\left(\mathrm{CH}_{2}\right), 43.63\left(\mathrm{CH}_{2}\right), 45.46\left(\mathrm{CH}_{2}\right), 110.68,118.68,124.75,125.11,129.22$, 129.36, 129.42, 129.87, 133.76, 133.95, 138.13, 140.69, 141.76, 148.98, 151.67, 155.66, 159.60, 164.56, 165.54 (C=O). For $\mathrm{C}_{30} \mathrm{H}_{28} \mathrm{~N}_{6} \mathrm{O}_{2} \mathrm{~S}$ HRMS $(m / z):[\mathrm{M}+\mathrm{H}]^{+}$ calcd: 537.65 ; found 537.20 .

\section{1-[4-(5,6-Bis-(4-methylphenyl)-1,2,4-triazin-3-yl)piperazin-1-yl]-2- [(1H-benzothiazol-2-yl)thio]ethan-1-one (3):}

$74 \%$ yield; mp $130{ }^{\circ} \mathrm{C}$. IR $v_{\max }\left(\mathrm{cm}^{-1}\right): 1647(\mathrm{C}=\mathrm{O}), 1525-1379(\mathrm{C}=\mathrm{C}, \mathrm{C}=\mathrm{N}), 1238-$ 1001 (C-N). ${ }^{1} \mathrm{H}-\mathrm{NMR}\left(300 \mathrm{MHz}, \mathrm{DMSO}-d_{6}\right.$ ppm) $\delta 2.32$ (s, 6H, $\mathrm{CH}_{3}$ ), 3.69 (brs, $2 \mathrm{H}$, piperazine $\mathrm{CH}_{2}$ ), 3.80 (brs, $2 \mathrm{H}$, piperazine $\mathrm{CH}_{2}$ ), 3.94 (brs, $2 \mathrm{H}$, piperazine $\mathrm{CH}_{2}$ ), 4.06 (brs, 2H, piperazine $\mathrm{CH}_{2}$ ), 4.64 (s, 2H, $\mathrm{CH}_{2} \mathrm{CO}$ ), 7.17 (d, J= $8.22 \mathrm{~Hz}$, $4 \mathrm{H}, \mathrm{Ar}-\mathrm{H}$ ), 7.27 (d, J=8.13 Hz, 2H, Ar-H), 7.34-7.7.40 (m, 3H, Ar-H), 7.46 (t, $\mathrm{J}=7.10 \mathrm{~Hz}, \mathrm{Ar}-\mathrm{H}$ ), 7.85 (d, J=7.83 Hz, 1H, Ar-H), 8.02 (d, J=7.86 Hz, 1H, Ar-H). ${ }^{13} \mathrm{C}-\mathrm{NMR}\left(75 \mathrm{MHz}, \mathrm{DMSO}-d_{6}, \mathrm{ppm}\right) \delta 21.29\left(\mathrm{CH}_{3}\right), 21.38\left(\mathrm{CH}_{3}\right), 37.14\left(\mathrm{COCH}_{2}\right)$, $41.89\left(\mathrm{CH}_{2}\right), 43.41\left(\mathrm{CH}_{2}\right), 43.71\left(\mathrm{CH}_{2}\right), 45.55\left(\mathrm{CH}_{2}\right), 121.52,122.29,124.93$, 126.84, 129.21, 129.36, 129.42, 129.88, 133.75, 133.96, 135.20, 138.13, 140.70, 148.97, 153.09, 155.64, 159.60, 165.75, $166.69(\mathrm{C}=\mathrm{O})$. For $\mathrm{C}_{30} \mathrm{H}_{28} \mathrm{~N}_{6} \mathrm{OS}_{2} \mathrm{HRMS}$ $(\mathrm{m} / \mathrm{z}):[\mathrm{M}+\mathrm{H}]^{+}$calcd: 553.72 ; found 553.18 .

\section{1-[4-(5,6-Bis-(4-methoxyphenyl)-1,2,4-triazin-3-yl)piperazin-1-yl]-2- [(1H-benzimidazol-2-yl)thio]ethan-1-one (4):}

78 \% yield; mp $243^{\circ} \mathrm{C}$. IR $v_{\max }\left(\mathrm{cm}^{-1}\right): 1647(\mathrm{C}=\mathrm{O}), 1608-1325(\mathrm{C}=\mathrm{C}, \mathrm{C}=\mathrm{N})$, 1244- $^{-}$ 1024 (C-N). ${ }^{1} \mathrm{H}-\mathrm{NMR}$ (300 MHz, DMSO- $d_{6}$ ppm) $\delta 3.67$ (brs, 2H, piperazine $\mathrm{CH}_{2}$ ), 3.77 (brs, $8 \mathrm{H}, \mathrm{OCH}_{3}$ and piperazine $\mathrm{CH}_{2}$ ), 3.91 (brs, $2 \mathrm{H}$, piperazine $\mathrm{CH}_{2}$ ), 4.03 (brs, 2H, piperazine $\mathrm{CH}_{2}$ ), 4.50 (s, 2H, $\mathrm{CH}_{2} \mathrm{CO}$ ), 6.91-6.96 (m, 4H, Ar-H), 7.10-7.13 (m, 2H, Ar-H), 7.32 (d, J= 8.79 Hz, 2H, Ar-H), 7.44-7.48 (m, 4H, Ar$\mathrm{H}), 12.57$ (s, $1 \mathrm{H}, \mathrm{NH}) .{ }^{13} \mathrm{C}-\mathrm{NMR}\left(75 \mathrm{MHz}, \mathrm{DMSO}-d_{6}, \mathrm{ppm}\right) \delta 35.58\left(\mathrm{COCH}_{2}\right)$, $41.79\left(\mathrm{CH}_{2}\right), 43.39\left(\mathrm{CH}_{2}\right), 43.70\left(\mathrm{CH}_{2}\right), 45.55\left(\mathrm{CH}_{2}\right), 55.61\left(\mathrm{OCH}_{3}\right), 55.79\left(\mathrm{OCH}_{3}\right)$, $114.25,114.34,121.85,128.63,129.26,130.57,131.64,148.65,150.22,154.98$, 159.52, 159.73, 161.45, 166.46. For $\mathrm{C}_{30} \mathrm{H}_{29} \mathrm{~N}_{7} \mathrm{O}_{3} \mathrm{~S}$ HRMS $(\mathrm{m} / \mathrm{z}):[\mathrm{M}+\mathrm{H}]^{+}$calcd: 568.67 ; found 568.21 .

\section{1-[4-(5,6-Bis-(4-methoxyphenyl)-1,2,4-triazin-3-yl)piperazin-1-yl]-2- [(1H-benzoxazol-2-yl)thio]ethan-1-one (5):}

78 \% yield; mp $215{ }^{\circ} \mathrm{C}$. IR $v_{\max }\left(\mathrm{cm}^{-1}\right): 1649(\mathrm{C}=\mathrm{O}), 1529-1377(\mathrm{C}=\mathrm{C}, \mathrm{C}=\mathrm{N})$, 1244- $^{-}$ 1026 (C-N, C-O). ${ }^{1} \mathrm{H}-\mathrm{NMR}$ (300 MHz, DMSO- $d_{6,}$ ppm) $\delta 3.69$ (brs, $2 \mathrm{H}$, piperazine $\mathrm{CH}_{2}$ ), 3.78 (brs, $8 \mathrm{H}, \mathrm{OCH}_{3}$ and piperazine $\mathrm{CH}_{2}$ ), 3.92 (brs, $2 \mathrm{H}$, piperazine $\mathrm{CH}_{2}$ ), 4.04 (brs, $2 \mathrm{H}$, piperazine $\mathrm{CH}_{2}$ ), 4.66 (s, 2H, $\mathrm{CH}_{2} \mathrm{CO}$ ), 6.92-6.96 (m, $4 \mathrm{H}$, Ar-H), 7.30-7.35 (m, 4H, Ar-H), 7.47 (d, J= 8.85 Hz, 2H, Ar-H), 7.63-7.68 (m, $2 \mathrm{H}, \mathrm{Ar}-\mathrm{H}) .{ }^{13} \mathrm{C}-\mathrm{NMR}\left(75 \mathrm{MHz}, \mathrm{DMSO}-d_{6}, \mathrm{ppm}\right) \delta 36.96\left(\mathrm{COCH}_{2}\right), 41.88\left(\mathrm{CH}_{2}\right)$, 
$43.69\left(\mathrm{CH}_{2}\right), 45.44\left(\mathrm{CH}_{2}\right), 55.80\left(\mathrm{OCH}_{3}\right), 110.68,114.26,114.34,118.67,124.75$, $125.11,128.63,129.25,130.57,131.64,148.67,155.0,159.51,159.74,161.46$, $165.54(\mathrm{C}=\mathrm{O})$. For $\mathrm{C}_{30} \mathrm{H}_{28} \mathrm{~N}_{6} \mathrm{O}_{4} \mathrm{~S}$ HRMS $(m / z)$ : $[\mathrm{M}+\mathrm{H}]^{+}$calcd: 569.65; found 569.20 .

1-[4-(5,6-Bis-(4-methoxyphenyl)-1,2,4-triazin-3-yl)piperazin-1-yl]-2[(1H-benzothiazol-2-yl)thio]ethan-1-one (6):

$71 \%$ yield; mp $189^{\circ} \mathrm{C}$. IR $v_{\max }\left(\mathrm{cm}^{-1}\right): 1647(\mathrm{C}=\mathrm{O}), 1527-1377(\mathrm{C}=\mathrm{C}, \mathrm{C}=\mathrm{N})$, 1244- $^{-}$ $1008(\mathrm{C}-\mathrm{N}) .{ }^{1} \mathrm{H}-\mathrm{NMR}$ (300 MHz, DMSO- $d_{6}$, ppm) $\delta 3.68$ (brs, $2 \mathrm{H}$, piperazine $\mathrm{CH}_{2}$ ), 3.77 (brs, $8 \mathrm{H}, \mathrm{OCH}_{3}$ and piperazine $\mathrm{CH}_{2}$ ), 3.93 (brs, $2 \mathrm{H}$, piperazine $\mathrm{CH}_{2}$ ), 4.04 (brs, $2 \mathrm{H}$, piperazine $\mathrm{CH}_{2}$ ), 4.63 (s, 2H, $\mathrm{CH}_{2} \mathrm{CO}$ ), 6.91-6.96 (m, 4H, Ar-H), 7.31-7.49 (m, 6H, Ar-H), 7.84 (d, J= 7.80 Hz, 2H, Ar-H), 8.02 (d, J=7.32 Hz, 1H, Ar-H). ${ }^{13} \mathrm{C}-\mathrm{NMR}\left(75 \mathrm{MHz}, \mathrm{DMSO}-d_{6}, \mathrm{ppm}\right) \delta 37.14\left(\mathrm{COCH}_{2}\right), 41.88\left(\mathrm{CH}_{2}\right), 43.41$ $\left(\mathrm{CH}_{2}\right), 43.71\left(\mathrm{CH}_{2}\right), 45.59\left(\mathrm{CH}_{2}\right), 55.61\left(\mathrm{OCH}_{3}\right), 55.80\left(\mathrm{OCH}_{3}\right), 114.25,114.34$, 121.52, 122.29, 124.93, 126.84, 128.63, 129.25, 130.57, 131.64, 148.67, 153.09, $154.98,159.52,159.74,161.46,165.74,166.69(\mathrm{C}=\mathrm{O})$. For $\mathrm{C}_{30} \mathrm{H}_{28} \mathrm{~N}_{6} \mathrm{O}_{3} \mathrm{~S}_{2}$ HRMS $(\mathrm{m} / \mathrm{z}):[\mathrm{M}+\mathrm{H}]^{+}$calcd: 585.21 ; found 585.17 .

\section{1-[4-(5,6-Bis-(4-chlorophenyl)-1,2,4-triazin-3-yl)piperazin-1-yl]-2- [(1H-benzimidazol-2-yl)thio]ethan-1-one (7):}

$78 \%$ yield; mp $175^{\circ} \mathrm{C} . \mathrm{IR} v_{\max }\left(\mathrm{cm}^{-1}\right): 1647(\mathrm{C}=\mathrm{O}), 1525-1379(\mathrm{C}=\mathrm{C}, \mathrm{C}=\mathrm{N}), 1240-$ $1014(\mathrm{C}-\mathrm{N}) .{ }^{1} \mathrm{H}-\mathrm{NMR}$ (300 MHz, DMSO-d $d_{6}$ ppm) $\delta 3.67$ (brs, 2H, piperazine $\mathrm{CH}_{2}$ ), 3.79 (brs, $2 \mathrm{H}$, piperazine $\mathrm{CH}_{2}$ ), 3.94 (brs, $2 \mathrm{H}$, piperazine $\mathrm{CH}_{2}$ ), 4.06 (brs, $2 \mathrm{H}$, piperazine $\mathrm{CH}_{2}$ ), $4.5 \mathrm{O}\left(\mathrm{s}, 2 \mathrm{H}, \mathrm{CH}_{2} \mathrm{CO}\right.$ ), 7.10-7.13 (m, 2H, Ar-H), 7.39-7.50 (m, 10H, Ar-H), 12.61 (s, $1 \mathrm{H}, \mathrm{NH}) .{ }^{13} \mathrm{C}-\mathrm{NMR}$ (75 MHz, DMSO-d $\left.d_{6} \mathrm{ppm}\right) \delta 35.56$ $\left(\mathrm{COCH}_{2}\right), 43.41\left(\mathrm{CH}_{2}\right), 43.72\left(\mathrm{CH}_{2}\right), 45.51\left(\mathrm{CH}_{2}\right), 122.03,129.02,131.19,131.83$, 133.74, 135.23, 135.32, 135.80, 147.80, 150.19, 154.93, 159.63, 166.49 (C=O). For $\mathrm{C}_{28} \mathrm{H}_{23} \mathrm{Cl}_{2} \mathrm{~N}_{7} \mathrm{OS} \operatorname{HRMS}(\mathrm{m} / \mathrm{z})$ : [M+H $]^{+}$calcd: 577.5O; found 577.11.

\section{1-[4-(5,6-Bis-(4-chlorophenyl)-1,2,4-triazin-3-yl)piperazin-1-yl]-2- [(1H-benzoxazol-2-yl)thio]ethan-1-one (8):}

$78 \%$ yield; mp $242{ }^{\circ} \mathrm{C}$. IR $v_{\max }\left(\mathrm{cm}^{-1}\right): 1651(\mathrm{C}=\mathrm{O}), 1527-1379(\mathrm{C}=\mathrm{C}, \mathrm{C}=\mathrm{N})$, 1240-1014 (C-N). ${ }^{1} \mathrm{H}-\mathrm{NMR}$ (300 MHz, DMSO- $d_{6}$ ppm) $\delta 3.69$ (brs, 2H, piperazine $\mathrm{CH}_{2}$ ), 3.78 (brs, $2 \mathrm{H}$, piperazine19 $\mathrm{CH}_{2}$ ), 3.95 (brs, $2 \mathrm{H}$, piperazine $\mathrm{CH}_{2}$ ), 4.07 (brs, $2 \mathrm{H}$, piperazine $\mathrm{CH}_{2}$ ), 4.66 (s, 2H, $\left.\mathrm{CH}_{2} \mathrm{CO}\right), 7.31-7.35(\mathrm{~m}, 2 \mathrm{H}, \mathrm{Ar}-\mathrm{H})$, 7.39-7.42 (m, 4H, Ar-H), 7.46-7.49 (m, 4H, Ar-H), 7.64-7.66 (m, 2H, Ar-H). ${ }^{13} \mathrm{C}-\mathrm{NMR}\left(75 \mathrm{MHz}, \mathrm{DMSO}-d_{6} \mathrm{ppm}\right) \delta 36.95\left(\mathrm{COCH}_{2}\right), 41.84\left(\mathrm{CH}_{2}\right), 43.39\left(\mathrm{CH}_{2}\right)$, 45.30 $\left(\mathrm{CH}_{2}\right)$, 110.68, 118.67, 124.75, 125.12, 129.0, 131.19, 131.82, 133.75, 135.22, $135.31,135.81,141.76,147.83,159.62,165.58(\mathrm{C}=\mathrm{O})$. For $\mathrm{C}_{28} \mathrm{H}_{22} \mathrm{Cl}_{2} \mathrm{~N}_{6} \mathrm{O}_{2} \mathrm{~S}$ HRMS $(\mathrm{m} / \mathrm{z}):[\mathrm{M}+\mathrm{H}]^{+}$calcd: 578.48 ; found 578.10 . 


\section{1-[4-(5,6-Bis-(4-chlorophenyl)-1,2,4-triazin-3-yl)piperazin-1-yl]-2- [(1H-benzothiazol-2-yl)thio]ethan-1-one (9):}

$78 \%$ yield; mp $124^{\circ} \mathrm{C}$. IR $v_{\max }\left(\mathrm{cm}^{-1}\right): 1647(\mathrm{C}=\mathrm{O}), 1525-1379(\mathrm{C}=\mathrm{C}, \mathrm{C}=\mathrm{N}), 1240-$ $1014(\mathrm{C}-\mathrm{N}) .{ }^{1} \mathrm{H}-\mathrm{NMR}\left(300 \mathrm{MHz}, \mathrm{DMSO}-d_{6}\right.$ ppm) $\delta 3.69$ (brs, $2 \mathrm{H}$, piperazine $\mathrm{CH}_{2}$ ), 3.80 (brs, $2 \mathrm{H}$, piperazine $\mathrm{CH}_{2}$ ), 3.95 (brs, $2 \mathrm{H}$, piperazine $\mathrm{CH}_{2}$ ), 4.07 (brs, $2 \mathrm{H}$, piperazine $\mathrm{CH}_{2}$ ), $4.64\left(\mathrm{~s}, 2 \mathrm{H}, \mathrm{CH}_{2} \mathrm{CO}\right.$ ), 7.36-7.39 (m, 2H, Ar-H), 7.427.49 (m, 1oH, Ar-H), 7.85 (d, J=7.56 Hz, 1H, Ar-H), 8.02 (d, J=8.25 Hz, 1H, Ar-H). ${ }^{13} \mathrm{C}-\mathrm{NMR}$ (75 MHz, DMSO- $\left.d_{6,} \mathrm{ppm}\right) \delta 37.12\left(\mathrm{COCH}_{2}\right), 41.84\left(\mathrm{CH}_{2}\right), 43.42$ $\left(\mathrm{CH}_{2}\right), 43.76\left(\mathrm{CH}_{2}\right), 45.49\left(\mathrm{CH}_{2}\right), 121.53,121.69,122.3 \mathrm{O}, 124.94,126.85,129.0$, 131.19, 131.83, 133.75, 135.32, 135.82, 147.82, 154.92, 159.63, 165.78 (C=O). For $\mathrm{C}_{28} \mathrm{H}_{22} \mathrm{Cl}_{2} \mathrm{~N}_{6} \mathrm{OS}_{2}$ HRMS $(\mathrm{m} / \mathrm{z}):[\mathrm{M}+\mathrm{H}]^{+}$calcd: 594.55; found 594.07.

\section{Biochemistry}

Cytotoxicity

For measuring the cytotoxic activity of compounds, MTT method (tetrazolium salt (3-(4,5-dimethylthiazol-2-yl)-2,5-diphenyltetrazolium bromide) was applied on MCF-7 breast adenocarcinoma and C6 rat glioma cell lines. The instruction in literature was followed to carry out experiments. To produce the cells, cell lines incubated in 96 well-plates for $24 \mathrm{~h}$ at $37^{\circ} \mathrm{C}$. Then, $20 \mu \mathrm{L}$ of MTT solution (5 mg/mL MTT in PBS) was added for each well and incubation was continued for more 2 hours at $37^{\circ} \mathrm{C}^{16,17}$. Before measuring the absorbance by ELISA reader (OD $570 \mathrm{~nm})$, dissolving of crystals using DMSO $(200 \mu \mathrm{L})$ was needed. Absorbance values were read and percentage of survival cells was calculated compared medium. All measurements were measured triplicate ${ }^{18}$.

In vitro lipoxygenase (LOX) inhibition assay

LOX inhibition activity was measured by a modified the spectrophotometric method developed by Baylac and Racine ${ }^{19}$. LOX (1.13.11.12, type I-B, Soybean), linoleic acid and all required chemicals were purchased from Sigma-Aldrich. Potassium phosphate buffer (1,94 mL; 100mM; $\mathrm{pH} 9.0), 40 \mu \mathrm{L}$ of test compound solution and $20 \mu \mathrm{L}$ of lipoxygenase solution were mixed and incubated for 10 min at $25{ }^{\circ} \mathrm{C}$. The reaction was then initiated by the addition of $10 \mu \mathrm{L}$ linoleic acid solution, the change of absorbance at $234 \mathrm{~nm}$ was followed for $10 \mathrm{~min}$. Test compounds and positive control Nordihydroguaiaretic acid (NDGA) were dissolved in methanol. All spectrum measurements were carried out in quartz cuvette avoiding material absorbance interference.

The concentration of test compounds which provided $50 \%$ inhibition $\left(\mathrm{IC}_{50}\right)$ of LOX enzyme was calculated and the experiments were performed triplicate for each concentration. For eliminating faults and non-enzymatic hydrolysis, blanks 
were compared without test compounds. Alteration in absorbance was recorded comparingly between with and without test compounds for determining enzyme inhibition activity and the results were given in percentages and standard deviations (SD+) were performed using Microsoft Office Excel 2013 program. ${ }^{20}$

\section{RESULTS AND DISCUSSION}

\section{Chemistry}

The synthesis of title compounds were carried out by a multi-step synthetic procedure according to previously reported study of us ${ }^{10}$. New 1-[4-(5,6-bis-(4substituted phenyl)-1,2,4-triazin-3-yl)piperazin-1-yl]-2-[(1H-(benz)imidazol/ thiazole/oxazole-2-yl)thio]ethan-1-one derivatives (1-9) were acquired with the reaction of 2-mercapto(benz)imidazole/thiazole/oxazoles and the intermediates (IIIa-c) which were already synthesized in early step. Nine final compounds were yielded in a range of $72 \%-78 \%$ range and melting points were found between $124^{\circ} \mathrm{C}$ and $248^{\circ} \mathrm{C}$.

In IR spectra of the compounds characteristic streching bands were observed at $1647-1680 \mathrm{~cm}^{-1}, 1325-1608 \mathrm{~cm}^{-1}$ and 1001-1269 $\mathrm{cm}^{-1}$ belong to $\mathrm{C}=\mathrm{O}$ double bond, $\mathrm{C}=\mathrm{C}$ and $\mathrm{C}=\mathrm{N}$ double bonds and $\mathrm{C}-\mathrm{N}$ and $\mathrm{C}-\mathrm{O}$ single bonds. According to ${ }^{1} \mathrm{H}-\mathrm{NMR}$ results, four methylene groups of piperazine rings resonated as broad singlets at about $3.67-4.07 \mathrm{ppm}$. The other methylene protons vicinal to carbonyl group were observed with a chemical shift at 4.50-4.68 ppm range. The aromatic protons of heterocyclic ring and phenyl rings were seen in between 7.0-8.80 $\mathrm{ppm}$. In the ${ }^{13} \mathrm{C}-\mathrm{NMR}$ spectra of the compounds, signals belonging to aliphatic carbon atoms were assigned at about $21.28-55.80 \mathrm{ppm}$; and signals for aromatic carbon atoms were observed at 101.68-165.75 ppm. The carbonyl carbon of amide group was seen at about $166 \mathrm{ppm}$. In mass spectroscopy, $[\mathrm{M}+\mathrm{H}]^{+}$peaks were established in accordance with the molecular weights of the compounds.

\section{Biology}

The cytotoxic activity of nine triazine compounds (1-9) were determined against C6 rat glioma and MCF-7 breast adenocarcinoma cell lines and results were summarized in Table 1. $\mathrm{IC}_{50}$ values were calculated in between $9.5-500 \mu \mathrm{g} / \mathrm{mL}$. Compound $4\left(\mathrm{IC}_{50}=21.0 \mu \mathrm{g} / \mathrm{mL}\right)$ was found as the most active compound even more than cisplatin against $\mathrm{C} 6$ cell line. Compound $\mathbf{6}$ was also exhibited good antiproliferative activity with a $\mathrm{IC}_{50}$ value of $26.0 \mu \mathrm{g} / \mathrm{mL}$ which is very close to cisplatin $\left(\mathrm{IC}_{50}=23.5 \mu \mathrm{g} / \mathrm{mL}\right)$. The $\mathrm{IC}_{50}$ values could not be calculated for compounds $\mathbf{1}, \mathbf{3}, \mathbf{8}$ and $\mathbf{9}$ even if at the highest tested concentration which is $500 \mu \mathrm{g} /$ $\mathrm{mL}$ against $\mathrm{C} 6$ tumor cells. Against MCF-7 cell line, compound $\mathbf{5}$ showed the strongest cytotoxicity $\left(\mathrm{IC}_{50}=9.5 \mu \mathrm{g} / \mathrm{mL}\right.$ ) which was higher than cisplatin. Com- 
pound $\mathbf{2}$ and $\mathbf{8}$ also showed good activity with $\mathrm{IC}_{50}$ values of 58.33 and $51.67 \mu \mathrm{g} /$ $\mathrm{mL}$, respectively. Two active compounds against C6 cells, compound 4 and $\mathbf{6}$ did not exhibite enough cytotoxic activity against MCF-7 cell line. Moreover, the lipooxygenase (LOX) inhibitory activity of the compounds were studied. None of the compounds showed LOX inhibition as much as standard drug nordihydroguaiaretic acid, even if $\mathrm{IC}_{50}$, the half maximal inhibitory concentration could not be calculated. At $100 \mu \mathrm{g} / \mathrm{mL}$ concentration, LOX inhibition percentages were identified for compounds $\mathbf{1}$ and $\mathbf{6}$ as $43.35 \%$ and $38.79 \%$, respectively. The results revelaed that 1,2,4-triazine derivatives have considerable cytotoxic activity. As a follow up study from past to present studies, antiproliferative activity of the compounds are rational and it worths to design new compounds based on this substructure.

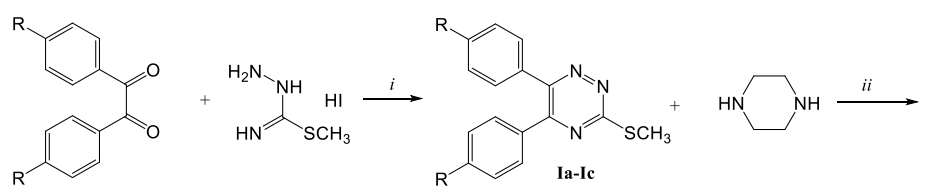

$\mathbf{R}=\mathrm{CH}_{3}, \mathrm{OCH}_{3}, \mathrm{Cl}$
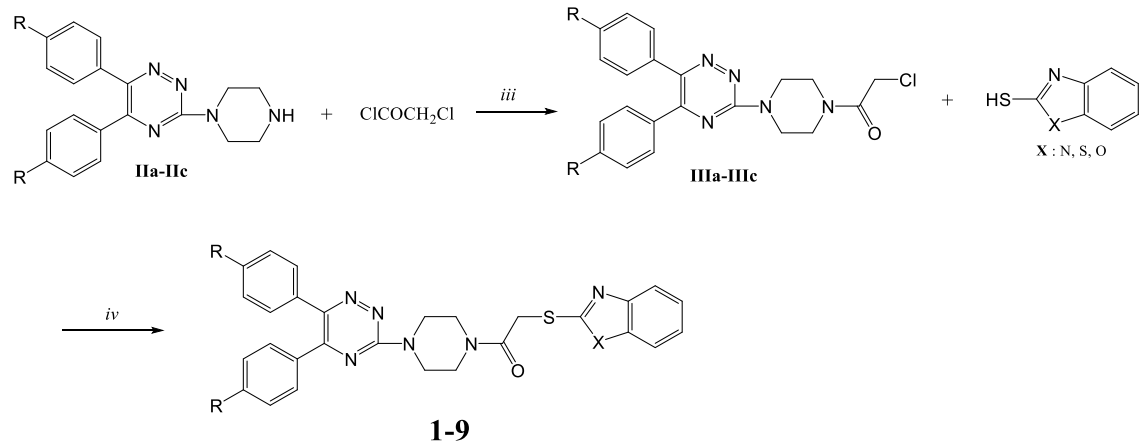

Scheme 1: The synthesis of the compounds. Reactants and reagents ; $i$. $\mathrm{NaHCO}_{3}, \mathrm{CH}_{3} \mathrm{OH}$, reflux $3 \mathrm{~h}$; ii: pyridine, reflux 6 h; iii: $\mathrm{Et}_{3} \mathrm{~N}$, DMF, r.t., $45 \mathrm{~min}$; $i v$. $\mathrm{K}_{2} \mathrm{CO}_{3}$, Acetone, r.t., $5 \mathrm{~h}$. 
Table 1: $\mathrm{IC}_{50}$ values $(\mu \mathrm{g} / \mathrm{mL})$ against $\mathrm{C} 6$ and MCF-7 tumor cell lines and \% LOX enzyme inhibition of the compounds

\begin{tabular}{cccc}
\hline Comp. & C6 & MCF-7 & $\begin{array}{c}\% \text { LOX inhb. (100 } \\
\mu \mathrm{g} / \mathrm{mL})\end{array}$ \\
\hline $\mathbf{1}$ & $>500$ & $>500$ & $43.35 \pm 3.08$ \\
$\mathbf{2}$ & $150.0 \pm 26.46$ & $58.33 \pm 2.89$ & -- \\
$\mathbf{3}$ & $>500$ & $>500$ & --- \\
$\mathbf{4}$ & $21.0 \pm 3.61$ & $>500$ & --- \\
$\mathbf{5}$ & $490.0 \pm 14.14$ & $9.5 \pm 0.50$ & --- \\
$\mathbf{6}$ & $26.0 \pm 1.73$ & $85.0 \pm 5.0$ & $38.79 \pm 0.86$ \\
$\mathbf{7}$ & $445.0 \pm 7.07$ & $>500$ & -- \\
$\mathbf{8}$ & $>500$ & $51.67 \pm 10.41$ & --- \\
$\mathbf{9}$ & $>500$ & $>500$ & --- \\
Cisplatin & $23.5 \pm 2.12$ & $11.67 \pm 2.89$ & -- \\
NDGA & - & - & $3.35 \pm 0.07$ \\
\hline
\end{tabular}

Not determined : ---

*NDGA : Nordihydroguaiaretic acid, $\mathrm{IC}_{50}$ value is represented in the corresponding line.

\section{REFERENCES}

1. Cancer Facts/Figures 2016, American Cancer Society. http://www.cancer.org/research/cancerfactsstatistics/cancerfactsfigures2016/index/ (Accessed August 22, 2016).

2. Wilson, C. O.; Gisvold, O.; and Doerge, R. F., Wilson and Gisvold's Textbook of Organic Medicinal and Pharmaceutical Chemistry. Philadelphia: Lippincott, 1982.

3. Montgomery, J.A. Studies on the Biologic Activity of Purine and Pyrimidine Analogs, Med. Res. Rev. 1982, 2, 271-308.

4. Liu, Y.; Laufer, R.; Patel, N.K.; Ng, G.; Sampson, P.B.; Li, S.W.; Lang, Y.; Feher, M.; Brokx, R.; Beletskaya, I.; Hodgson, R.; Plotnikova, O.; Awrey, D.E.; Qiu, W.; Chirgadze, N.Y.; Mason, J.M.; Wei, X.; Lin, D.C.; Che, Y.; Kiarash, R.; Fletcher, G.C.; Mak, T.W.; Bray, M.R.; Pauls, H.W. Discovery of Pyrazolo[1,5-a]pyrimidine TTK Inhibitors: CFI-4O2257 is a Potent, Selective, Bioavailable Anticancer Agent. ACS Med. Chem. Lett. 2016, 6, 671-675.

5. Singla, P.; Luxami, V.; Paul, K. Synthesis and In Vitro Evaluation of Novel Triazine Analogues As Anticancer Agents and Their Interaction Studies with Bovine Serum Albumin. Eur J Med Chem. 2016, 19, 59-69.

6. Krauth, F.; Dahse, H.M.; Rüttinger, H.H.; Frohberg, P. Synthesis and Characterization of Novel 1,2,4-Triazine Derivatives with Antiproliferative Activity. Bioorg Med Chem. 2010, 18, 1816-1821.

7. Al-Issa, S.A. Synthesis and Anticancer Activity of Some Fused Pyrimidines and Related Heterocycles. Saudi Pharm. J. 2013, 21, 305-316.

8. Sztanke, K.; Pasternak, K.; Rzymowska, J.; Sztanke, M.; Kandefer-Szerszeń, M. Synthesis, Structure Elucidation and Identification of Antitumoural Properties of Novel Fused 1,2,4-Triazine Aryl Derivatives. Eur. J. Med. Chem. 2008, 43, 1085-1094. 
9. Hunt, J.T.; Mitt, T.; Borzilleri, R.; Gullo-Brown, J.; Fargnoli , J.; Fink, B.; Han, W.C.; Mortillo, S.; Vite, G.; Wautlet, B.; Wong, T.; Yu, C.; Zheng, X.; Bhide, R. Discovery of the Pyrrolo $[2,1-f][1,2,4]$ triazine Nucleus As a New Kinase Inhibitor Template. J. Med. Chem. 2oo4, 47, 4054-4059.

10. Walters, T.; Aur R.J., Hernandez K. 6-Azauridine in Combination Chemotherapy of Childhood Acute Myelocytic Leukemia. Cancer 1972, 29, 1057-1060.

11. Abd El-All, A.S.; Osman, S.A.; Roaiah, H.M.F.; Abdalla, M.M.; Abd El Aty, A.A.; Abd ElHady, W.H. Potent Anticancer and Antimicrobial Activities of Pyrazole, Oxazole and Pyridine Derivatives Containing 1,2,4-Triazine Moiety. Med. Chem. Res. 2015, 24, 4093-4104.

12. El-Gendy, Z.; Morsy, J.M.; Allimony, H.A.; Abdel-Monem, W.R.; Abdel-Rahman, R.M. Synthesis of Heterobicyclic Nitrogen Systems Bearing A 1,2,4-Triazine Moiety as Anticancer Drugs: Part IV. Phosphorus, Sulfur 2003, 178, 2055-2071.

13. De Lucia, D.; Lucio, O.M.; Musio, B.; Bender, A.; Listing, M.; Dennhardt, S.; Koeberle, A.; Garscha, U.; Rizzo, R.; Manfredini, S.; Werz, O.; Ley, S.V. Design, Synthesis and Evaluation of Semi-synthetic Triazole-containing Caffeic Acid Analogues As 5-Lipoxygenase Inhibitors. Eur. J. Med. Chem. 2015, 101, 573-583.

14. Cai, H.; Huang, X., Xu, S.; Shen, H.; Zhang, P.; Huang, Y.; Jiang, J.; Sun, Y., Jiang, B.; Wu, X.; Yao, H.; Xu, J. Discovery of Novel Hybrids of Diaryl-1,2,4-Triazoles and Caffeic Acid As Dual Inhibitors of Cyclooxygenase-2 and 5-Lipoxygenase For Cancer Therapy. Eur. J. Med. Chem. 2016, 108, 89-103.

15. Yurttas, L.; Demirayak, S.; Ilgın, S.; Atlı, Ö. In Vitro Antitumor Activity Evaluation of Some 1,2,4-Triazine Derivatives Bearing Piperazine Amide Moiety Against Breast Cancer Cells, Bioorg. Med. Chem. 2014, 22, 6313-6323.

16. Mossmann, T. Rapid Colorimetric Assay for Cellular Growth and Survival: Application to Proliferation and Cytotoxicity Assays. J. Immunol. Methods. 1983, 16, 55-63.

17. Keiser, K.; Johnson, C.C.; Tipton, D.A. Cytotoxicity of Mineral Trioxide Aggregate Using Human Periodontal Ligament Fibroblasts. J. Endod. 200o, 26, 288-291.

18. Yurttaş, L.; Demirayak, Ş.; Akalın Çiftçi, G. Cytotoxic, Antiproliferative and Apoptotic Effects of New Benzimidazole Derivatives on A549 Lung Carcinoma and C6 Glioma Cell Lines. Anticancer Agents Med. Chem. 2015, 15, 1174-1184.

19. Baylac, S.; Racine, P. Inhibition of 5-Lipoxygenase by Essential Oils and Other Natural Fragrant Extracts. Int. J. Aromather. 2003, 13, 138-142.

20. Demirci, B.; Temel, H.E.; Portakal, T.; Kurmızıbekmez, H.; Demirci, F.; Baser, K.H.C. Inhibitory Effect of Calamintha nepeta subsp. Glandulosa Essential Oil on Lipoxygenase. Turk $J$. Biochem. 2011, 36, 290-295.

(Received o6 September 2016; accepted 26 September 2016) 